\title{
Differentiation of Schwann-like cells from human umbilical cord blood mesenchymal stem cells in vitro
}

\author{
YU-ZHOU XIAO ${ }^{1,2^{*}}$ and SHENG WANG ${ }^{3 *}$ \\ ${ }^{1}$ Department of Orthopedics; ${ }^{2}$ Anhui Key Laboratory of Tissue Transplantation, \\ The First Affiliated Hospital of Bengbu Medical College; \\ ${ }^{3}$ Department of Orthopedics, The Second Affiliated Hospital of Bengbu Medical College, \\ Bengbu, Anhui 233004, P.R. China
}

Received December 2, 2013; Accepted August 8, 2014

DOI: $10.3892 / \mathrm{mmr} .2014 .2840$

\begin{abstract}
The use of artificial nerves for the repair of peripheral nerve defects is restricted by the limited sources of Schwann cells (SCs). Human mesenchymal stem cell (MSC)-derived Schwann-like cells are considered an alternative and desirable cell source. The aim of the present study was to establish a method of inducing directional differentiation of human umbilical cord blood (hUCB) MSCs into Schwann-like cells. Cells isolated from hUCB were cultured in MesenCult complete medium, a specialized culture medium for MSCs, to expand hUCBMSCs. hUCBMSCs were purified by repeated changing of the medium, and they were identified by detection of the specific cell surface markers for MSCs. For differentiation of Schwann-like cells from hUCBMSCs, the purified cells were sequentially cultured in DMEM/F12 medium with various additives. Differentiated Schwann-like cells were identified by the detection of SC-specific markers, including S100b, glial fibrillary acidic protein and P75, by immunocytochemisty, reverse transcription-polymerase chain reaction and western blotting. The results demonstrated that the majority of the differentiated cells presented classical dipolar and fusiform SC morphology. Notably, a large proportion of these cells expressed the three SC markers. These results suggest that hUCBMSCs can undergo directional differentiation into Schwann-like cells in vitro and may be an important source of SCs for the treatment of peripheral nerve defects with tissue-engineered artificial nerves.
\end{abstract}

Correspondence to: Professor Yu-Zhou Xiao, Department of Orthopedics, The First Affiliated Hospital of Bengbu Medical College, 287 Chang Huai Road, Bengbu, Anhui 233004, P.R. China E-mail: xiaoyuzhou_2013@163.com

*Contributed equally

Key words: umbilical cord blood, mesenchymal stem cell, schwann cell, differentiation

\section{Introduction}

The seed cells of artificial nerves are Schwann cells (SCs), however, the limited availability of these cells has been a persistent challenge to clinical application. Stem cells can be used as a source of seed cells as they lead to a reduced probability of immunological rejection, and have been successfully used in nerve tissue regeneration. A study by Heath (1) demonstrated that the use of stem cells as seed cells is able to improve regeneration and increase the compatibility of transplanted tissue with adjacent tissue. Due to their low immunogenicity, stem cells may reduce or even eliminate the requirement for immunosuppressive drugs.

Human bone marrow mesenchymal stem cells (MSCs) differentiated into Schwann-like cells have been used as artificial nerve seed cells, utilizing the sciatic nerve lesion model in rats (2-4). However, another study indicated that the content and ability of multidirectional differentiation of bone marrow cells reduces with increasing age of the donor, posing limitations on the use of this treatment in older patients (5). In contrast, MSCs isolated from human umbilical cord blood (hUCB) are not affected by donor age. Furthermore, hUCB has been widely investigated due to its vast source options, easy and atraumatic collection, low immunogenicity (6) and high differentiation capability. In the current study, the differentiation of hUCBMSCs into Schwann-like cells in vitro was investigated under specific induction conditions.

\section{Materials and methods}

Umbilical cord blood sample. Umbilical cord blood was provided by the Department of Obstetrics of the First Affiliated Hospital of Bengbu Medical College (Bengbu, China). The patients and their family members provided signed informed consent documents prior to the study. This study was performed with the approval of the ethical committee of the First Affiliated Hospital of Bengbu Medical College (Bengbu, China; no. 2012029).

Instruments and reagents. The following equipment was used in the proceeding experiments: Refrigerated horizontal centrifuge 5810 R (Eppendorf, Hamburg, Germany); $\mathrm{CO}_{2}$ 
incubator (Thermo Fisher Scientific, Waltham, MA, USA); clean bench (Suzhou Purification Engineering Installation Co., Ltd., Suzhou, China); inverted microscope (CKX41SF; Olympus Corp., Tokyo, Japan); BD FACSCalibur Flow Cytometer (BD Biosciences, Franklin Lakes, NJ, USA); gene amplification apparatus (Black King Kong EDC-810, Dongsheng Chuangxin Biotechnology Co., Ltd, Beijing, China); nucleoprotein detector (BioPhotometer plus; Eppendorf AG, Hamburg, Germany) and a Tanon 3500 Gel Imaging System (Tanon Science and Technology Co., Ltd., Shanghai, China). The following reagents were used: MesenCult Proliferation Kit (Human) (05411; Stemcell Technologies, Inc., Vancouver, Canada); hespan 6\% hetastarch in $0.9 \%$ sodium chloride injection (B Braun Medical, Inc., Bethlehem, PA, USA); fetal bovine serum (FBS) and Dulbecco's modified Eagle's medium (DMEM)/F12 (Hyclone Laboratories, Logan UT, USA); lymphocyte separation media (human) ( $\rho=1.077 \mathrm{~g} / \mathrm{ml}$ ) (Haoyang Biological Manufacture Co., Ltd, Tianjin, China); pancreatin, $\beta$-sodium glycerophosphate and 3-isobutyl-1-methylxanthine (IBMX) (Sigma-Aldrich, St. Louis, MO, USA); anti-CD34-fluorescein isothiocyanate (FITC), CD44-phycoerythrin (PE) and CD73-PE (BioLegend, Inc., San Diego, CA, USA); $\beta$-mercaptoethanol ( $\beta$-ME; Amresco LLC, Solon, OH, USA); retinoic acid (RA; \#R2625; Sigma-Aldrich); recombinant human fibroblast growth factor-basic (bFGF; \#100-18B), recombinant human platelet-derived growth factor- $B B$ (PDGF-BB; \#100-14B), recombinant human heregulin $\beta-1$ (HRG; \#100-03) and recombinant human $\beta$-nerve growth factor (NGF; \#450-01) (all from PeproTech, Inc., Rocky Hill, NJ, USA); forskolin (FSK; \#S1612; Beyotime Institute of Biotechnology, Shanghai, China); anti-S100 (Maixin Biotech Co., Ltd, Fuzhou, China); rabbit anti-S100b (Beijing Biosynthesis Biotechnology Co., Ltd., Beijing, China); mouse anti-GFAP (Maixin Biotech Co., Ltd.); mouse anti-GFAP (\#AG259; Beyotime Institute of Biotechnology); rabbit anti-P75 (Beijing Biosynthesis Biotechnology Co., Ltd.); E.Z.N.A. Total RNA Kit I (\#R6834-00; Omega Bio-Tek, Inc., Norcross, GA, USA); RevertAid reverse transcriptase (\#EP0441; Thermo Fisher Scientific, Pittsburgh, PA, USA), PCR Master Mix (2X) (\#K1621; Thermo Fisher Scientific); and non-biotin immunohistochemical Elivision ${ }^{\mathrm{TM}}$ plus kit (Maixin Biotech Co., Ltd).

Isolation and cultivation of hUCBMSCs. A volume of 40-60 ml umbilical cord blood from full-term normal delivery or cesarian-section fetuses were collected under aseptic conditions and separated $6 \mathrm{~h}$, subsequent to anticoagulation, with heparin. Umbilical cord blood was mixed with $6 \%$ hetastarch at a volume ratio of 1:4 and incubated at room temperature for $45 \mathrm{~min}$. The supernatant was collected and added to human lymphocyte separation media (volume ratio of 1:2). The mononuclear cell suspension containing hUCBMSCs was obtained from the cloudy white interphase following centrifugation for $20 \mathrm{~min}$ at $690 \mathrm{xg}$ at room temperature. DMEM/F12 was added to the cells (volume ratio of 1:5), and the cells were then washed twice with DMEM/F12 and counted with a hematocytometer. The hUCBMSCs were resuspended in MesenCult complete medium (from the proliferation kit) at $1.0-1.5 \times 10^{7}$ cells $/ \mathrm{ml}$, seeded into a $25 \mathrm{~cm}^{2}$ plastic culture flask, and cultured at $37^{\circ} \mathrm{C}$ in a $5 \%$ $\mathrm{CO}_{2}$ humidified incubator. The cell culture media was changed daily for the 5-7 days after seeding and then changed every 3 days. Cells of $90 \%$ confluence were digested by $0.25 \%$ trypsin (Sigma-Aldrich). The morphological changes in the cultured cells were observed using an inverted microscope.

hUCBMSC differentiation to osteocytes and adipocytes. Osteogenic differentiation. Cells at passage 3 (P3) at a density of $5 \times 10^{4} / \mathrm{cm}^{2}$ were added to a six-well plate and incubated in osteogenesis-inducing medium, consisting of DMEM/F12 containing $10 \mathrm{mM} \beta$-sodium glycerophosphate, $0.1 \mu \mathrm{M}$ dexamethasone, $50 \mu \mathrm{g} / \mathrm{ml}$ vitamin-C (all Sigma-Aldrich) and 10\% FBS. Cells in the control group were not treated with this media. The cell culture media was changed every three days. The cells were cultured for 21 days and then stained with $0.1 \%$ alizarin red (Sigma-Aldrich) for calcium nodule staining.

Adipogenic differentiation. Cells at P3 were seeded into a 6-well plate in adipogenesis-inducing medium, consisting of $\mathrm{DMEM} / \mathrm{F} 12$ containing $1 \mu \mathrm{M}$ dexamethasone, $10 \mu \mathrm{g} / \mathrm{ml}$ insulin, $0.5 \mathrm{mM}$ IBMX, $0.1 \mu \mathrm{M}$ indomethacin (all Sigma-Aldrich), and $10 \%$ FBS. Control cells were not treated with this media. The culture media was changed every 3 days. After 21 days, the cells were stained with $2 \%$ oil red O staining solution (Sigma-Aldrich).

Directional differentiation of hUCBMSCs to SCs. P3 cells were seeded onto $0.01 \%$ poly-L-lysine (Sigma-Aldrich)-coated coverslips placed in a six-well plate. When the adherent cells grew to $60-70 \%$ confluence, differentiation was induced. First, cells were pre-induced with DMEM/F12 containing $0.25 \mathrm{mM} \beta$-ME and $10 \mathrm{ng} / \mathrm{ml} \mathrm{bFGF}$ for $24 \mathrm{~h}$. The culture media was then changed to DMEM/F12 with $10 \%$ FBS. Following $24 \mathrm{~h}$ incubation, the culture media was changed to DMEM/F12 supplemented with $35 \mathrm{ng} / \mathrm{ml} \mathrm{RA}$ for $24 \mathrm{~h}$. The culture media was then changed to DMEM/F12 containing 10\% FBS. Subsequent to another $24 \mathrm{~h}$ incubation, the cells were incubated in DMEM/F12 supplemented with $5 \mu \mathrm{M}$ FSK, $10 \mathrm{ng} / \mathrm{ml}$ bFGF, $5 \mathrm{ng} / \mathrm{ml}$ PDGF-BB, $100 \mathrm{ng} / \mathrm{ml} \mathrm{NGF}$ and $200 \mathrm{ng} / \mathrm{ml} \mathrm{HRG}$ for 4 days. The control cells were cultured in DMEM/F12 containing 10\% FBS. The cell morphology was observed using an inverted microscope.

Immunocytochemistry. Cells grown on glass coverslips were fixed with $4 \%$ paraformaldehyde for $30 \mathrm{~min}$ and washed with PBS ( $\mathrm{pH} 7.4$ ) 3 times, for 3 min each. The fixed cells were incubated in $3 \%$ hydrogen peroxide solution for 10 min to block endogenous catalase, washed with PBS, and then incubated with the anti-S100, GFAP and P75 primary antibodies for $60 \mathrm{~min}$ at room temperature. The cells were washed 3 times with PBS, incubated for $20 \mathrm{~min}$ in polymer reinforcing agent (agent A from the Elivision kit), washed with PBS, and then incubated for $30 \mathrm{~min}$ in enzyme-labeled resistant rat/rabbit polymer (agent B). The slides were developed with diaminobenzidine (Maixin Biotech Co., Ltd, Fuzhou, China) and counterstained with hematoxylin. The samples were examined using an inverted microscope.

Reverse transcription $(R T)$ and polymerase chain reaction $(P C R)$. Total RNA was extracted with the E.Z.N.A. Total RNA Kit, cDNA was synthesized with the RevertAid reverse transcriptase and the product was amplified using PCR Master Mix (2X). The primers were synthesized by Sangon Biotech Co., Ltd. (Shanghai, China) (Table I). The PCR cycling parameters 
Table I. Primer sequences and expected product sizes following polymerase chain reaction.

\begin{tabular}{lllr}
\hline Gene & Direction & \multicolumn{1}{c}{ Sequence } & Size (bp) \\
\hline S100b & Sense & 5'-GGAAATCAAAGAGCAGGAGGT-3' & 254 \\
& Antisense & 5'-ATTAGCTACAACACGGCTGGA-3' & 406 \\
GFAP & Sense & 5'-GTCCATGTGGAGCTTGACG-3' & 371 \\
& Antisense & 5'-CATTGAGCAGGTCCTGGTAC-3' & \\
P75 & Sense & 5'-TGGACAGCGTGACGTTCTCC-3' & 546 \\
& Antisense & 5'-GATCTCCTCGCACTCGGCGT-3' & \\
& Sense & 5'-GGGACCTGACTGACTACCTC-3' & \\
& Antisense & 5'-ACTCGTCATACTCCTGCTTGCTG-3' &
\end{tabular}

bp, base pairs.
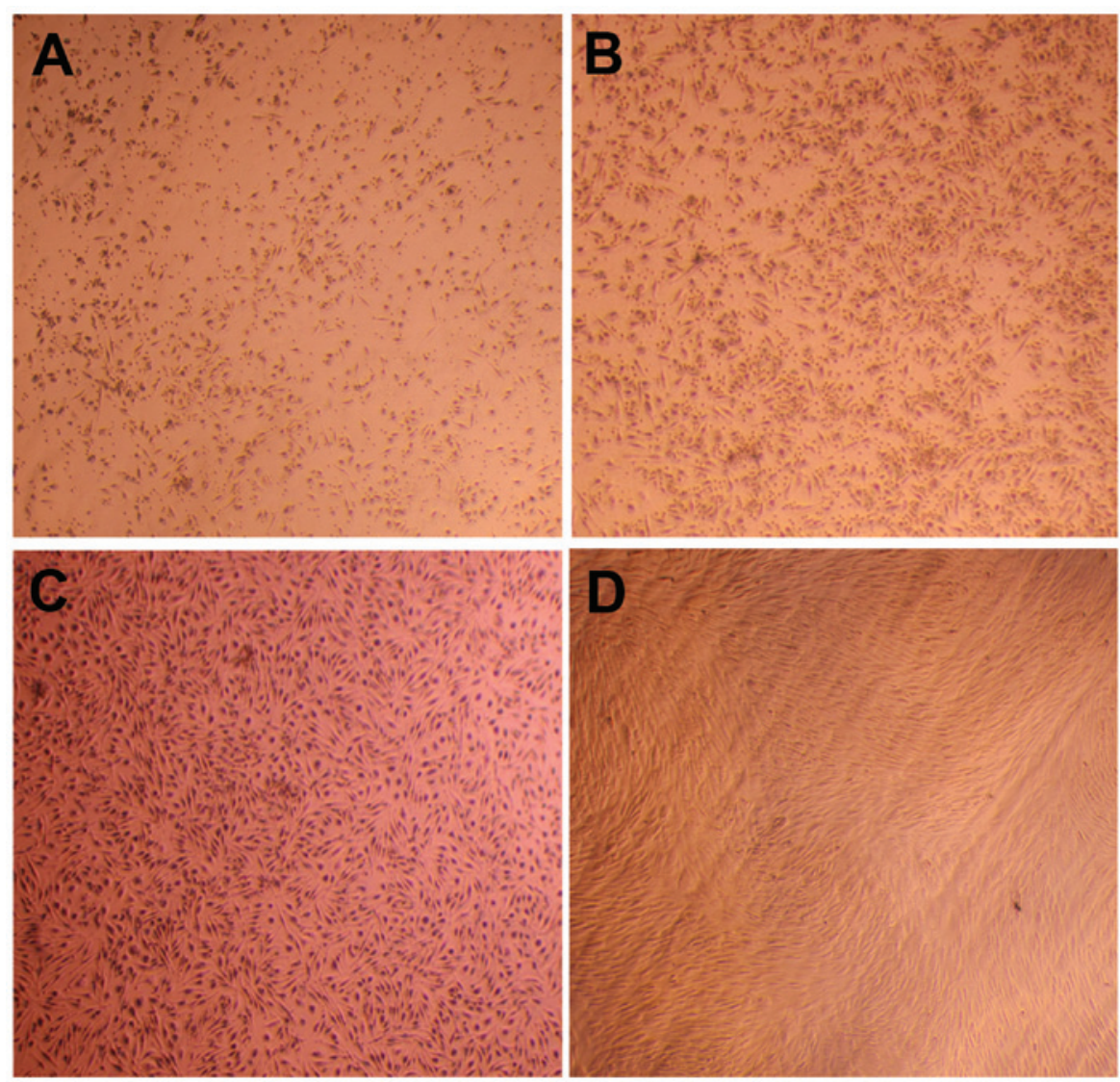

Figure 1. Morphology of human umbilical cord blood mesenchymal stem cells. (A) Adherent cells following 72-h culture. (B) An increased number of attached cells were observed following a medium change after 5 days in cluture. (C) Confluence reached $80 \%$ after $\sim 3$ weeks in primary culture. (D) The third passage cells are uniformly long-spindle shaped with spiral or parallel growth. Magnification, x40.

were as follows: Initial denaturation step $\left(95^{\circ} \mathrm{C}, 3 \mathrm{~min}\right)$ followed by 35 cycles of denaturation $\left(95^{\circ} \mathrm{C}, 30 \mathrm{sec}\right)$, annealing $(30 \mathrm{sec}$; S100b, $54.4^{\circ} \mathrm{C}$; GFAP, $59.9^{\circ} \mathrm{C} ; \mathrm{P} 75,59.9^{\circ} \mathrm{C} ; \beta$-actin, $55.6^{\circ} \mathrm{C}$ ) and primer extension $\left(72^{\circ} \mathrm{C}, 45 \mathrm{sec}\right)$ followed by final extension incubation $\left(72^{\circ} \mathrm{C}, 10 \mathrm{~min}\right)$. The products were analyzed using a gel imaging system following 1.5\% agarose gel electrophoresis (100V, $25 \mathrm{~min})$.

Western blotting. The cultured cells were washed with PBS and lysed with a lysis buffer $(50 \mathrm{mM}$ Tris- $\mathrm{HCl}, 150 \mathrm{mM}$
$\mathrm{NaCl}$ buffer, $1 \%$ NP-40, $0.5 \%$ sodium deoxycholate, $0.1 \%$ SDS, $1 \mathrm{mM}$ EDTA, $1 \mathrm{mM}$ sodium orthovanadate, $10 \mathrm{mM}$ sodium fluoride, $4 \mu \mathrm{g} / \mathrm{ml}$ leupeptin, $1 \mu \mathrm{g} / \mathrm{ml}$ aprotinin and $100 \mu \mathrm{g} / \mathrm{ml}$ PMSF; all from Sigma-Aldrich), and the total protein was extracted. The concentration of the total protein was measured using an enhanced bicinchoninic acid protein assay kit (Beyotime Biotech, Shanghai, China). The proteins were denatured by boiling for $5 \mathrm{~min}$. A total of $45 \mu \mathrm{g}$ protein was separated by $12 \%$ SDS-PAGE (Sigma-Aldrich) and transferred to a nitrocellulose (NC) membrane (EMD Millipore, 

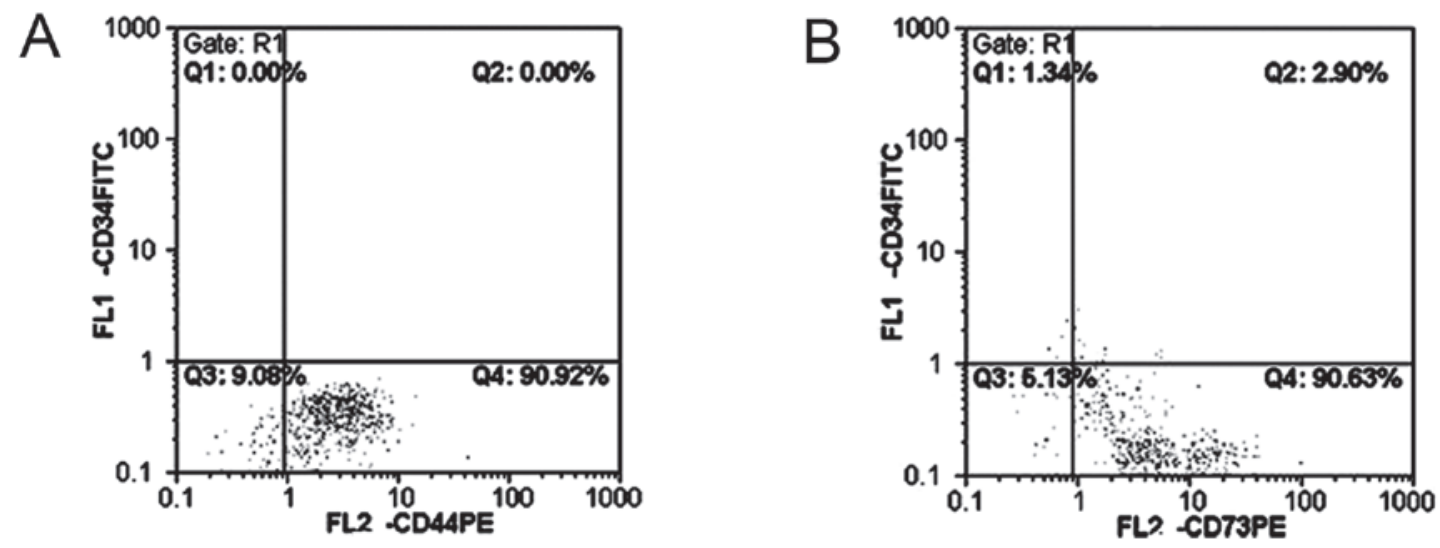

Figure 2. Fluorescence-activated cell sorting analysis of hUCBMSCs. (A) Expression of stem cell adhesion molecule CD44 in cultured hUCBMSCs. (B) Expression of stem cell surface antigen CD73 in the cultured hUCBMSCs. hUCBMSCs, human umbilical cord blood mesenchymal stem cells; FITC, fluorescein isothiocyanate.
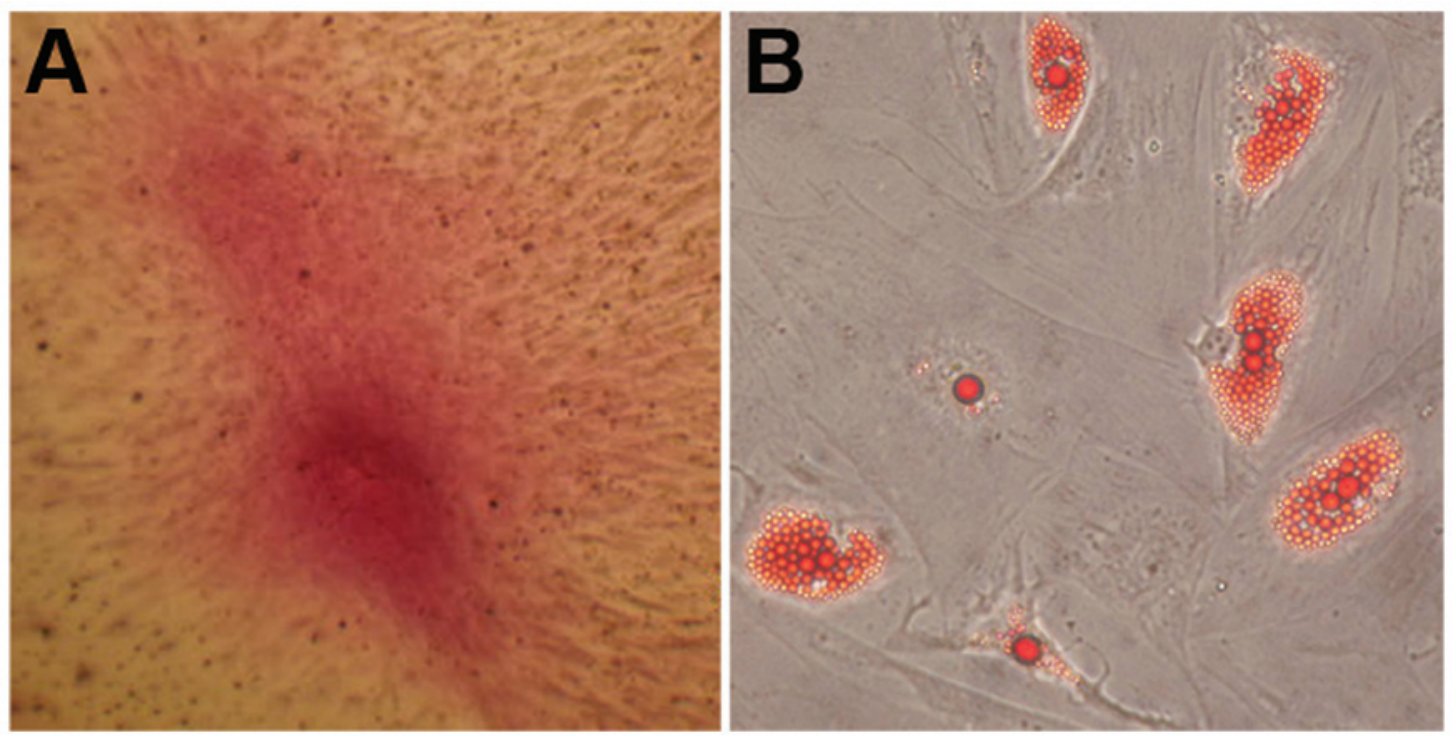

Figure 3. Differentiation of osteocytes and adipocytes. (A) Red cell with calcifying nodules dyed with alizarin red following osteogenic differentiation (magnification, $x$ 40). (B) Cardinal red lipid droplets in the lipocyte hyaloplasm stained with oil red $\mathrm{O}$ following adipogenic differentiation (magnification, x100).

Billerica, MA, USA). The NC membranes were blocked with $5 \%$ skimmed milk prior to incubation with the primary antibodies at $37^{\circ} \mathrm{C}$ for $1 \mathrm{~h}$ followed by overnight incubation at $4^{\circ} \mathrm{C}$. The primary antibodies used were those against S100b (1:200), GFAP (1:300) and P75 (1:200). The membranes were incubated with secondary antibodies goat anti-rabbit $\operatorname{IgG}(1: 6,000)$ and goat anti-mouse $\operatorname{IgG}(1: 6,000)$ and then exposed using Luminata Crescendo premixed horseradish peroxidase chemiluminescence substrate (EMD Millipore).

\section{Results}

Isolation and culture of hUCBMSCs in vitro. The mononuclear cells of the primary passage were seeded in a plastic culture flask supplemented with MesenCult complete medium. After $72 \mathrm{~h}$, a small number of cells adhered to the plastic culture flask (Fig. 1A). After five days, numerous adherent cells were observed, the majority of which were a short-fusiform shape and evenly distributed (Fig. 1B). A number of round-shaped osteoclast-like cells were also observed. With increased culture time, the cell number increased and colony formation occurred. Following two weeks of culturing, the number of mixed round-shaped cells was substantially reduced, while that of the short fusiform-shaped cells increased, and the morphology changed into a long-fusiform shape. Following 3 weeks, when the cell confluence reached $90 \%$, the cells were similar to fibroblast cells, with a uniformly long-fusiform shape and smaller size (Fig. 1C). Nearly all other types of cells were absent and only the uniformly fibroblast-like hUCBMSCs remained upon subculturing at the third passage (Fig. 1D). The cells were broad and flat and appeared similar to bone marrow MSCs. A number of the cells began to present signs of aging and the rate of amplification reduced when subcultured to the seventh passage.

Identification of surface markers by flow cytometry. hUCBMSCs exhibited a lack or reduced expression of hematopoietic stem/progenitor cell antigen CD34, and highly expressed the MSC surface antigens CD44 and CD73 (Fig. 2). 

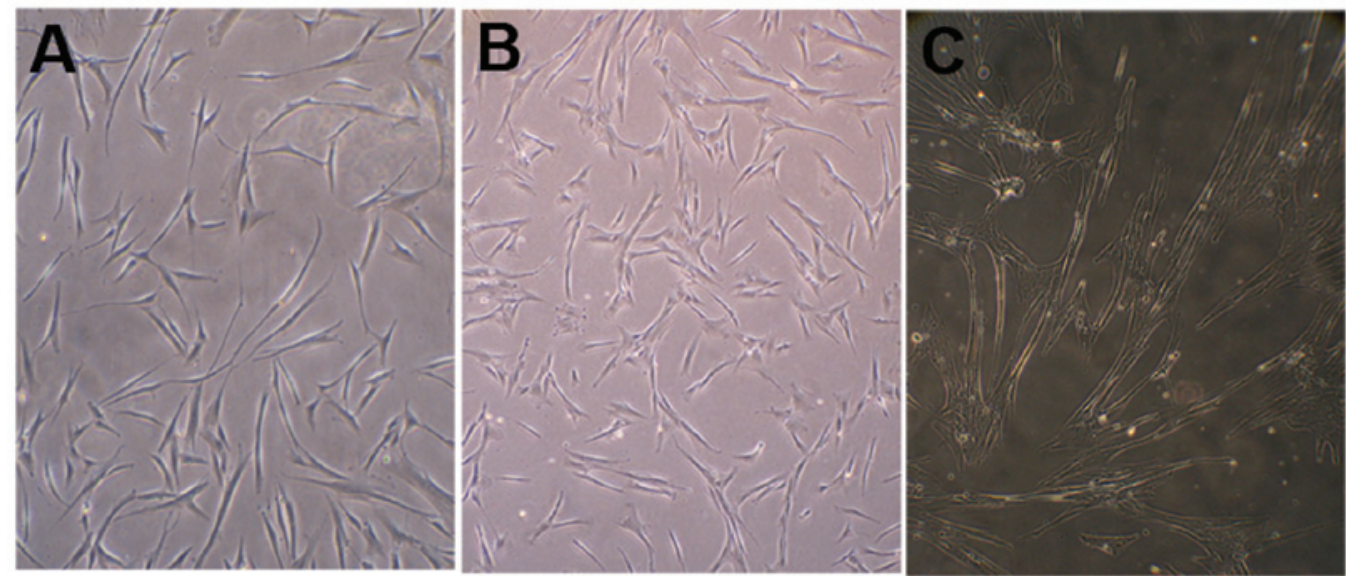

Figure 4. Morphological changes subsequent to directionally-induced differentiation of hUCBMSCs to Schwann-like cells. (A) The cell bodies contracted and the edges became irregular following pre-induction by $\beta$-ME+bFGF (magnification, $\mathrm{x} 40$ ). (B) The cell bodies contracted further, taking on an irregular conical or triangular morphology following induction with RA for $24 \mathrm{~h}$ (magnification, $\mathrm{x} 40$ ). (C) The cells exhibited spindle-like or triangular morphology, with a number of them presenting protuberances subsequent to the completion of induction (magnification, x100). hUCBMSCs, human umbilical cord blood mesenchymal stem cells; RA, retinoic acid.
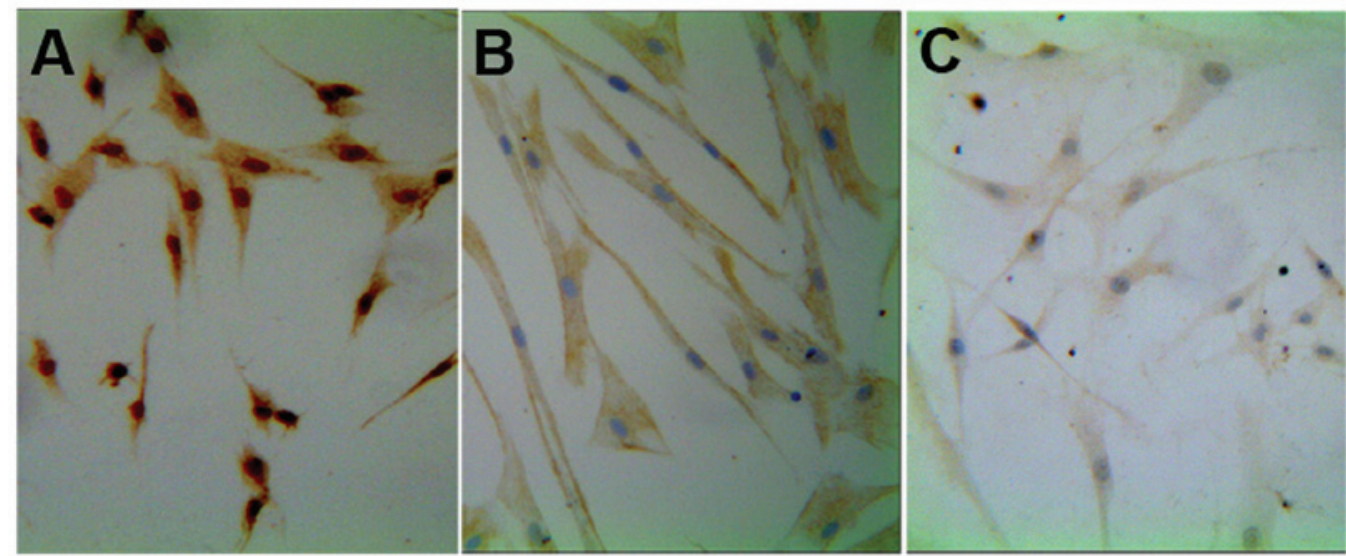

Figure 5. Differentiated cells were identified by immunocytochemisty. (A) Positive staining for the hyaloplasm/nucleus. (B) Positive staining for GFAP. (C) P75 hyaloplasm staining. Magnification, x100. GFAP, glial fibrillary acidic protein.

Differentiation of hUCBMSCs to osteocytes and adipocytes and their identification.

Osteogenic differentiation. One week after induction, the cells became $100 \%$ confluent and aggregated during culture. Clear thickening and nodosity in regions were observed following 2 weeks, and major red cell nodules were observed after three weeks following staining with alizarin red (Fig. 3A).

Adipogenic differentiation. Enlarged cells and tiny lipid droplets were observed in the hyaloplasm 1 week subsequent to induction. Highly refractive lipid droplets were formed in the hyaloplasm after 2 weeks. Following cultivation for 3 weeks, tiny lipid droplets increased in number and merged to form larger droplets filling the entire cell. The lipid droplets in the hyaloplasm turned red when stained with oil red O (Fig. 3B).

Morphological changes following directionally-induced differentiation from hUCBMSCs to Schwann-like cells. The cell body of the long-fusiform MSCs began retracting and the edge appearance became irregular following pre-induction with $\beta$-ME and bFGF (Fig. 4A). The edges retracted further after induction with RA and changed to an irregular conical or triangular shape (Fig. 4B). Following induction with medium containing FSK, bFGF, PDGF-BB, NGF and HRG, the frequency of the cell body contractions and the spaces between the cells increased. Subsequent to induction, the cell morphology altered to a slender spindle or triangular shape similar to Schwann cells (Fig. 4C).

Schwann-like cells express glial cell markers. Almost all of the differentiated cells expressed the glial cell markers S100b, GFAP and P75 (Fig. 5), the majority of which ( 70\%) exhibited the classical dipolar fusiform morphology of SCs. Glial cell marker gene transcripts were detected by RT followed by PCR (Fig. 6). Differentiated human (dh) MSCs expressed the transcripts for S100b (254 bp), GFAP (406 bp) and p75 (371 bp) whilst the undifferentiated human (uh) MSCs did not. Western blotting (Fig. 7) indicated positive protein expression for the glial proteins S100b (10 kDa), GFAP (50 Da) and p75 (75 kDa) in dhMSC, which were not expressed in uhMSCs. 


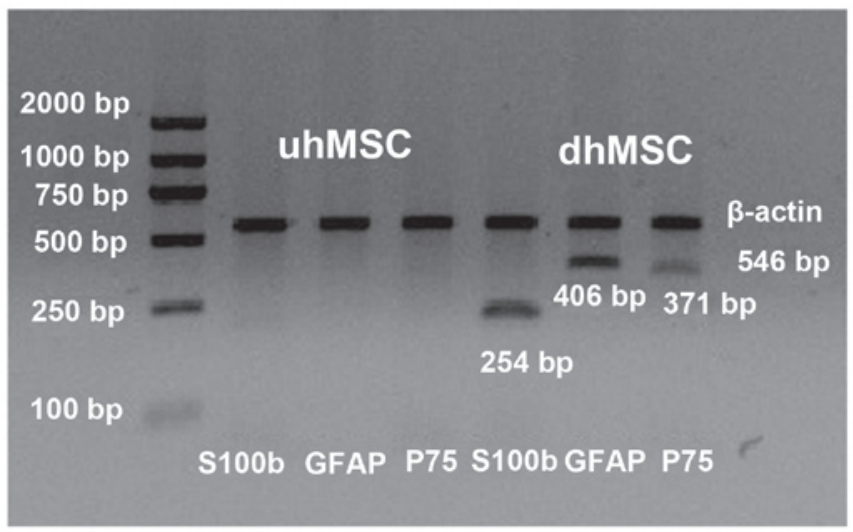

Figure 6. Polymerase chain reaction analysis indicated that dhMSC expressed S100b, GFAP and P75, whereas uhMSC were negative for these markers. $\beta$-actin served as an internal control. dhMSC, differentiated human mesenchymal stem cell; uhMSC, undifferentiated hMSC; bp, base pairs.

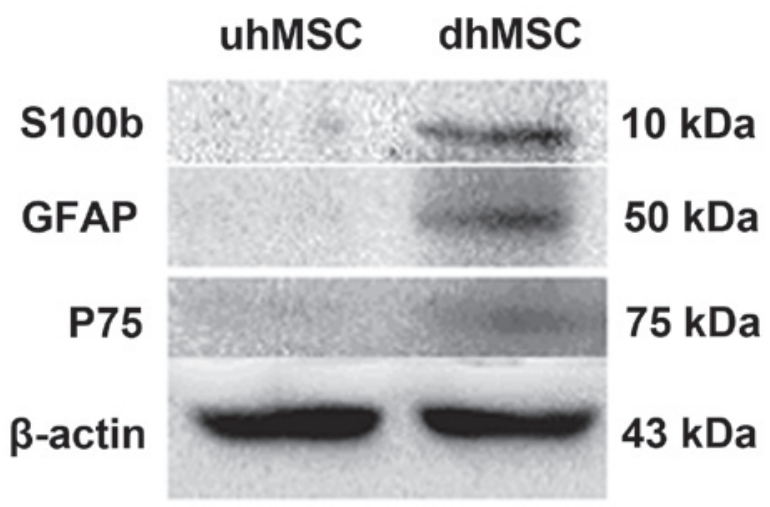

Figure 7. Western blotting revealed that dhMSCs, but not uhMSC, expressed $\mathrm{S} 100 \mathrm{~b}, \mathrm{GFAP}$ and $\mathrm{P} 75$ proteins. $\beta$-actin was used as an internal control. dhMSC, differentiated human mesenchymal stem cell; uhMSC, undifferentiated hMSC; kDa, kilodaltons.

\section{Discussion}

Erices et al (7) described the generation of fibroblast-like cells upon separation of umbilical cord blood. These cells expressed the MSC surface antigens SH2, SH3, SH4, ASMA, MAB1470, CD13, CD29 and CD49e, as do bone marrow MSCs. Lee et al (8) acquired MSCs following cord blood separation by gradient centrifugation. A rudimentary identification and description of these cells was provided, which further confirmed the presence of MSCs in UCB. In the current study, the plastic-adherent cells separated from UCB exhibited a lack of, or reduced expression, of hematopoietic stem/progenitor cell antigen CD34, and highly expressed the stem cell antigens CD44 and CD73. These results exclude the possibility of the presence of hematopoietic stem cells. It is possible for the cells to be differentiated to osteoblasts and adipocytes. According to the minimal criteria described for MSCs (9), the presence of hUCBMSCs can be confirmed by the results of the present study.

hUCBMSCs have become a potential substitution source of human bone marrow MSCs through tissue engineering in artificial nerve treatment of peripheral nerve defects. These cells have significant advantages in their clinical application prospects, as they are able to be induced to differentiate into glial cells. Lee et al (8) acquired GFAP-positive cells subsequent to generating hUCBMSCs with a multistage induction method. Zhang et al (10) then produced Schwann-like cells through improved methods of inducing hUCBMSC differentiation from embryonic stem cells. In the present study, hUCBMSC differentiation to Schwann-like cells was successfully induced, based on the methods of Dezawa et al (11) and Wang and Liu (4). However, notable modifications were made from their protocols with regards to the induction process. Intermittent time was included for $24 \mathrm{~h}$ following pre-induction by $\beta-\mathrm{ME}$ and $\mathrm{bFGF}$, the dosage of $\beta$-ME was reduced, and the induction time with RA was shortened. This approach improved cell viability and cytoactivity, and also reduced cell death by minimizing damage from chemical induction and allowing more time for the recovery of damaged cells. Additionally, the number of typical bipolar fusiform SCs increased dramatically subsequent to induction with NGF.

Immunocytochemistry analysis suggested that almost all the differentiated cells expressed glial cell markers, similar to the result of Tohill et al (12), who induced the differentiation of rat MSCs. In addition, the present study provided genetic and molecular evidence that these cells exhibit the characteristics of SCs, in addition to morphological similarity. The differentiation protocol of hUCBMSC to SC was investigated based on the preliminary understanding of differentiation mechanisms. During induction, $\beta$-ME activates cell surface channels or receptors directly, leading to cell contraction and phenotypic changes, including cytoplasmic neurite elongation and alteration of cytoskeletal structure within the cell (13). bFGF can accelerate the transition from SC precursors to SCs (14). A combination of $\beta$-ME and bFGF was used pre-induction to accelerate the transition from MSCs to SCs in the preliminary experiments. RA, derived from vitamin A, strongly induces differentiation, and its biological effect is mediated by the RA receptor (15). Generally, RA forms a complex with cell RA binding protein in the cytoplasm subsequent to entering cells. It then forms a complex with the chromatin receptor upon entering the nuclear core leading to alteration of cellular phenotype through regulation of specific gene expression (15). As potent mitogens, bFGF and PDGF are able to activate MAP kinase (MAPK) in MSCs to stimulate DNA synthesis in SCs (14). HRG and neuregulin, which are growth factors of the same family, are able to encourage SC proliferation. HRG is regarded as the pivotal signal that controls SC progression at each stage of the lineage $(14,16)$. It has been reported (17) that HRG is able to induce neural crest cell differentiation to SCs by activating MAPK signaling. FSK is commonly used to activate adenylate cyclase and elevate cAMP levels in cells (18). Additionally, FSK is able to increase the expression of growth factor receptors and, when combined with bPGF, PDGF-BB and HRG, enhances the synergism of SC differentiation (19).

NGF is a type of neurocyte growth regulatory factor that has dual biological functions in neuron nutrition and the promotion of neurite growth, and it is also able to enhance the metabolism of various types of neurocytes. NGF has the ability to protect and nourish normal neurocytes after maturation, to maintain the sensation of the pars affecta and subsistence of the sympathetic neurons, and to promote axon growth in order to repair damaged nerve fibers. In addition, NGF is able to promote the differentiation of neural precursor cells to mature neurons and glial cells 
in vitro (20). When bound to the TrkA receptor, NGF imparts its biological effect through the ERK/MAPK and PI3K/AKT signal transduction pathways, initiating a series of reactions that regulate structures on target cells or gene expression of functional proteins (21). However, co-culturing with DRGs does not support previous evidence that NGF can upregulate FSK (22). Regardless, in the present study, the number of induced cells presenting the classical morphology of SCs increased after adding NGF to the induction medium.

In conclusion, the current study established a modified method of inducing directional differentiation of hUCBMSCs to Schwann-like cells, and verified the morphological and phenotypic similarity between the induced cells with SCs. These data suggest that HUCBMSCs are able to proliferate substantially in vitro and also that they exhibit similar surface markers and a similar multidirectional differentiation potential to bone marrow MSCs. Future studies should aim to determine the physiological function of Schwann-like cells in vivo, providing experimental support for their application in the clinical repair of peripheral nerve defects through formation of tissue engineered artificial nerves on scaffold materials.

\section{Acknowledgements}

The current study was supported by the Key Medical Research Project of Department of Health of Anhui Province (2010A015).

\section{References}

1. Heath CA: Cells for tissue engineering. Trends Biotechnol 18 17-19, 2000.

2. Brohlin M, Mahay D, Novikov LN, et al: Characterisation of human mesenchymal stem cells following differentiation into Schwann cell-like cells. Neurosci Res 64: 41-49, 2009.

3. Shimizu S, Kitada M, Ishikawa H, Itokazu Y, et al: Peripheral nerve regeneration by the in vitro differentiated-human bone marrow stromal cells with Schwann cell property. Biochem Biophys Res Commun 359: 915-920, 2007.

4. Wang J and Liu K: Method of differentiation of adult human bone marrow mesenchymal stem cell into Schwann-like cells in vitro. Beijing Da Xue Xue Bao 35: 202-206, 2003 (In Chinese).

5. Sethe S, Scutt A and Stolzing A: Aging of mesenchymal stem cells. Ageing Res Rev 5: 91-116, 2006.
6. Wang M, Yang Y, Yang D, et al: The immunomodulatory activity of human umbilical cord blood-derived mesenchymal stem cells in vitro. Immunology 126: 220-232, 2009.

7. Erices A, Conget P and Minguell JJ: Mesenchymal progenitor cells in human umbilical cord blood. Br J Haematol 109: 235-242, 2000.

8. Lee OK, Kuo TK, Chen WM et al: Isolation of multipotent mesenchymal stem cells from umbilical cord blood. Blood 103: 1669-1675, 2004.

9. Dominici M, Le Blanc K, Mueller I, et al: Minimal criteria for defining multipotent mesenchymal stromal cells. The International Society for Cellular Therapy position statement. Cytotherapy 8: 315-317, 2006.

10. Zhang HT, Cheng HY, Zhang L, et al: Umbilical cord blood cell-derived neurospheres differentiate into Schwann-like cells. Neuroreport 20: 354-359, 2009.

11. Dezawa M, Takahashi I, Esaki M, et al: Sciatic nerve regeneration in rats induced by transplantation of in vitro differentiated bone-marrow stromal cells. Eur J Neurosci 14: 1771-1776, 2001.

12. Tohill M, Mantovani C, Wiberg M and Terenghi G: Rat bone marrow mesenchymal stem cells express glial markers and stimulate nerve regeneration. Neurosci Lett 362: 200-203, 2004.

13. Croft AP and Przyborski SA: Formation of neurons by non-neural adult stem cells: potential mechanism implicates an artifact of growth in culture. Stem Cells 24: 1841-1851, 2006.

14. Matsuse D, Kitada M, Kohama M, et al: Human umbilical cord-derived mesenchymal stromal cells differentiate into functional Schwann cells that sustain peripheral nerve regeneration. J Neuropathol Exp Neurol 69: 973-985, 2010.

15. Schmidt-Mende J, Gogvadze V, Hellström-Lindberg E and Zhivotovsky B: Early mitochondrial alterations in ATRA-induced cell death. Cell Death Differ 13: 119-128, 2006.

16. Xu Y, Liu Z, Liu L, et al: Neurospheres from rat adipose-derived stem cells could be induced into functional Schwann cell-like cells in vitro. BMC Neurosci 9: 21, 2008.

17. Ogata T, Yamamoto S, Nakamura K and Tanaka S: Signaling axis in schwann cell proliferation and differentiation. Mol Neurobiol 33: 51-62, 2006.

18. Jang S, Cho HH, Cho YB, et al: Functional neural differentiation of human adipose tissue-derived stem cells using bFGF and forskolin. BMC Cell Biol 11: 25, 2010.

19. Lin W, Chen X, Wang X, et al: Adult rat bone marrow stromal cells differentiate into Schwann cell-like cells in vitro. In vitro Cell Dev Biol Anim 44: 31-40, 2008.

20. Benoit BO, Savarese T, Joly M, et al: Neurotrophin channeling of neural progenitor cell differentiation. J Neurobiol 46: 265-280, 2001.

21. Song EJ and Yoo YS: Nerve growth factor-induced neurite outgrowth is potentiated by stabilization of TrkA receptors. BMB Rep 44: 182-186, 2011.

22. Mahay D, Terenghi G and Shawcross SG: Schwann cell mediated trophic effects by differentiated mesenchymal stem cells. Exp Cell Res 314: 2692-2701, 2008. 